It was considered proper to apprise the husband and sister of the patient of her present desperate conofion, and to inform them that the only probable though slight chance of escape rested either on the operation of gastrotcmy being performed, should the patient rally, or on leaving the case to the healing powers of nature. The relations immediately decided that no operation should be performed.

Although I thoroughly disagree with Dr. Denman in the following sentence, as a general rule, still, under the peculiar circumstances of this case, I was not sorry at the conclusion to which the relatives had arrived :-Speaking of interference in cases of rupture of the uterus, Dr. Denman says-" "When the uterus is ruptured at the time of labor, both reason and experience show that the patient has a better chance of recovery by resigning the case to the natural efforts of the constitution, than by any operation or interference of art." I am aware that Dr. Denman subsequently somewhat modified this opinion regarding cases where turning is inadmissible.

It was determined in the case under consideration to watch symptoms; should any opportunity of interfering with advantage occur, to seize it, in the mean time to support the strength with mild nourishment, and to administer opiates.

Wednesday, February 1. About two o'clock, a.m., the patient slept, and awoke refreshed. At two o'clock, p.m., she felt better; the skin was warm, and gentle perspiration covered it; the pulse was 120 , soft, and full. Six o'clock p.m.: Has had some sleep, and is more composed. Ten o'clock, p.m.: Pulse slower, full, and soft; a warm perspiration over the whole body; countenance free from anxiety.

Thursday, 2nd of February. Morning visit: Has slept composedly all night, and feels better. The husband stated his conviction that our prognosis had been too unfavorable. 'T'en o'clock, p.m.: 'The bad symptoms have returned; cold clammy sweat over the whole body ; the pulse was very quick. and small ; facies Hippocratica.

Friday, February 3. The patient gradually sank, and died about four o'clock, a.m., having survived the rupture about fifty hours.

With consent of the relations, I now made an incision, of about five inches in length, in the course of the linea alba. An immense quantity of fluid blood gushed out, covering the bed and floor. I introduced my right hand through the opening into the abdomen, and readily obtained hold of both feet, which, together with the body, I brought out at the external wound; I found, however, that I could not extract the head, as the incision was not long enough. Upon enlarging the opening, the head, enormously distended with fluid, was produced. The child, a male, was, as might be anticipated, dead.

The placenta came away easily; it appeared to have been expelled from the cavity of the uterus into the abdomen.

The dimensions of the head were as follows:-From the occipital protuberance to the nasal suture, sixteen and a half inches; the arch from the meatus auditorious externus of one side to that of the opposite, fourteen and a half inches; the circumference, twenty inches.

As I was anxious that some of my medical friends should be present at the examination of the body in the afternoon, I requested permission to reopen the abdomen, which, after some difficulty, was obtained.

The rupture in the uterus was to the right side posteriorly, and did not extend to the vagina; the uterus was contracted to about the size of a small infant's head; the peritoneum was of a dusky red color; the dimensions of the pelvis were natural.

The principal peculiarities in this case appear to be the fact of the rupture occurring before the os uteri was dilated by the uterine contractions, and its being occasioned apparently by the violent volnntary efforts of the patient to force forward the head of the infant, she being misled by the circumstance of the child's birth having in all her former labors immediately followed the rupture of the membranes, and having in consequence falsely concluded that the same succession of events must necessarily follow in this case. The hydrocephalic condition of the child, rendering the relative proportions of the head and pelvis so irregular, must, of course be taken into considera. tion.-Lond. and Edin. Monthly Journ.

\section{MEDICAL APPOINTMENTS}

WESTMINSTER HOSPITAL

Dr. Basham has been elected physician to this hos pital, a vacancy having been made by the resignation of Dr. Bright.

ST. PETER's hospital, BRISTOL.

Dr. William Budd and Dr. O'Brien, of Bristol, have just been elected physicians to St. Peter's Hospital, in that city, in the room of Drs. Lyon and Bernard, resigned.

\section{ROYAL COLLEGE OF SURGEONS IN LONDON.}

Members admitted Friday, July 21, 1843.

T. Cattell, J. C. Harper, G. M. Henning, H. F. Williams, P. P. Ransom, 'T. L. Philipps, A Markwick, R. D. Walker, J. L. Milton.

\section{ERRATA.}

In Mr. Husband's letter, at page 337 of our last Number, "Mr. Truman" was erroneously printed for Mr. Thurnam. The reader is also requested to correct the following errata in the same Number:Page 324, col. 1, lines 5.3 and 55, for "Dr. Taubert," read $M$. Jobert ; p. 332, col. 2, line 37, for "publish one," read publish it in one; p. 333 , col. 1 , line 27 , for "reach," read react ; line 32, for "liable," read loath; line 56, for "have," read take.

\section{B} EAMISH and CRAWFORD'S BAVARIAN PALE STOU'T.

This pure and wholesome Malt Liquor, brewed upon principles personally explained by PROFESSOR LIEBIG to the Manufacturers, is, by the peculiar mode adopted in its preparation, rendered a most desirable beverage to persons of delicate constitution, who are unable to use other malt liquors, however excellent of their kind, which are brewed according to the ordinary method; and from such persons numerous 'Testimonials have been received of the beneficial effect they have experienced from drinking it.

Beamish and Crawford's Bavarian Brown Stout and Extra Brown Stout sold in Bottles, by Falkner and Bancks, sole Consignees, 12, Villiers-street, Strand. Mr. W. Richardson, 1, Union-terrace, Notting-hill, Agent for Kensington, Hammersmith, and Acton. 